\title{
SULPHUR PARTICLES IMMOBILIZED ON ACTIVATED POLYSTYRENE SURFACE
}

\author{
'Simona LUPÍNKOVÁ, ${ }^{1}$ Jaroslava JAROLÍMKOVÁ, ${ }^{1}$ Martin KORMUNDA, ${ }^{1}$ Zdeňka KOLSKÁ \\ ${ }^{1} J$. E. Purkinje University in Usti nad Labem, Faculty of Science, Usti nad Labem, Czech Republic, EU, \\ simona.lupinkova@email.cz
}

https://doi.org/10.37904/nanocon.2020.3735

\begin{abstract}
Materials with immobilized or entrapped nanoparticles are of considerable interest. Sulphur as one of the abundant elements on earth is cheap and environmentally friendly. In this work, we have studied the surface properties of polystyrene foil and changes of its surface properties after (i) activation by ultraviolet radiation and (ii) subsequent grafting with sulphur particles (SPs). We have also investigated the effect of surface pregrafting with cysteamine on subsequent SPs grafting. SPs have been prepared by an acid catalyzed precipitation of sodium thiosulphate in the presence of sodium dodecyl sulphate as capping agent. The changes in surface properties of modified foils were characterized by contact angle measurement, electrokinetic analysis and X-ray photoelectron spectroscopy (XPS). The contact angle decreased after UV activation due to the formation of oxygen polar groups. The presence of sulphur and sodium on the polystyrene surface, revealed by XPS, showed that SPs were bound to this surface. The surface morphology of samples and grafted SPs size were examined by scanning electron microscopy. The significant changes in SPs agglomeration after grafting on surface were observed.
\end{abstract}

Keywords: Polystyrene, sulphur particles, surface modification, UV treatment, surface properties

\section{INTRODUCTION}

The modification of polymer surfaces by the covalent attachment of molecules, nanoparticles or microspheres has been an active topic of investigation in the field of surface sciences and has provided an opportunity to fabricate novel heterostructures with desired properties [1-3].

Sulphur is a widely used element in different applications such as fertilizers, pharmaceuticals, antimicrobial agents, insecticides and fumigants. Sulphur nano- and micro- particles have been examined for modification of metal and carbon nanotubes, synthesis of composites for lithium batteries, anti-cancer agent, antibacterial agent and as adsorbent for the extraction of metal ions [4,5].

The aim of our research was to bring an insight and understanding into the mechanism of UV activation of polymer surface and its subsequent functionalization with molecules and particles.

In this study, the sulphur particles (SPs) have been synthesized by quick precipitation method in the presence of sodium dodecyl sulphate (SDS) that was used as a stabilizer to control the SPs size. We have investigated size, size distribution and zeta potential of prepared particles by dynamic light scattering (DLS). We have also focused on the modification of polystyrene foil by UV radiation and subsequent grafting with stabilized sulphur particles (SDS-SPs). The surface properties (polarity, surface chemistry and structure) of polystyrene before and after modification were studied by various techniques (goniometry, electrokinetic analysis and X-ray photoelectron spectroscopy). Scanning electron microscopy was employed for imaging the microstructure and morphology of surface. 


\section{EXPERIMENTAL}

\subsection{Chemicals and materials}

The chemicals used for this study were taken from the following companies: sodium thiosulfate pentahydrate ( $\mathrm{Na}_{2} \mathrm{~S}_{2} \mathrm{O}_{3} \cdot 5 \mathrm{H}_{2} \mathrm{O}$, Lach:Ner, CZ), oxalic acid $\left(\mathrm{H}_{2} \mathrm{C}_{2} \mathrm{O}_{4} \cdot 2 \mathrm{H}_{2} \mathrm{O}\right.$, Lach:Ner, CZ), sodium dodecyl sulphate (SDS, Sigma-Aldrich, USA), cysteamine (cys, $\left.\mathrm{HS}(\mathrm{CH})_{2}\right)_{2} \mathrm{NH}_{2}, 98 \%$, Sigma Aldrich, USA).

Polystyrene foil (PS, thickness $50 \mu \mathrm{m}$, biaxially oriented, GoodFellow Ltd., UK) was used for the experiments.

\subsection{Particle synthesis}

SPs were synthesized according to the method described in the work [3], with slight modifications. A mixture of sodium thiosulfate and SDS was prepared by combining $45 \mathrm{ml}$ of $0.05 \mathrm{M} \mathrm{Na}_{2} \mathrm{~S}_{2} \mathrm{O}_{3}$ with $1 \mathrm{ml}$ of $0.05 \mathrm{M} \mathrm{SDS}$. The mixture was stirred mechanically at $350 \mathrm{rpm}$ at room temperature $\left(25^{\circ} \mathrm{C}\right)$. Then an appropriate amount of 0.3 Macid solution was added to the mixture under continuous stirring. A white precipitate was observed after a while; the reaction was stopped after $40 \mathrm{~min}$. The particles suspension was centrifuged and washed with distilled water. The obtained precipitate was dispersed in distilled water for subsequent modification of PS samples.

\subsection{Surface modification of polystyrene}

PS foil was activated by UV radiation for $15,30,45$ or 60 minutes using UV lamp with wavelength $254 \mathrm{~nm}$. Immediately after activation the samples were inserted into $1 \%$ aqueous solution of cysteamine. After 24 hours the samples were rinsed with distilled water and inserted to the SPs solution for 24 hours. After this time, samples were rinsed with distilled water and allowed to air dry.

\subsection{Samples characterization}

The zeta potential and the particle size of prepared SPs were determined by dynamic light scattering using LitesizerTM 500 (Anton Paar, AUT). Values were determined at room temperature, with a semiconductor laser, $40 \mathrm{~mW}, 658 \mathrm{~nm}$, at constant $\mathrm{pH}$ with repeatability of $10 \%$. The particles were analyzed immediately after being prepared.

Surface properties of the PS samples (pristine, activated, or grafted) were studied using the following methods:

Tensile strength test was used to evaluate mechanical properties of PS samples before and after UV activation using testing machine LabTest 6.050 (Labortech, CZ). Samples were cut in the longitudinal and transverse direction. The tensile strength was calculated from the maximum stretching force at break divided by the original cross-sectional area of the sample (width $\times$ thickness).

The surface morphology was investigated using scanning electron microscopy (SEM, VEGA, Tescan, CZ). Measurements were carried out using secondary electron imaging mode with acceleration voltage $20 \mathrm{kV}$. The samples were sputtered by a $20 \mathrm{~nm}$ thick gold layer to eliminate surface charging.

The contact angle characterizing surface wettability was determined by the sessile drop technique employing a goniometer DSA30 (KRÜSS GmbH, DE). Droplets of volume $(2.00 \pm 0.05) \mu \mathrm{l}$ were positioned at the centre of the surface of samples. The values of contact angle were evaluated automatically by ADVANCE software.

Zeta potential of pristine and modified PS samples was accomplished on SurPASS Instrument (Anton Paar, AUT). Samples were fixed on two sample holders (size $2 \times 1 \mathrm{~cm}^{2}$ and gap between $100 \mu \mathrm{m}$ ) and placed inside the adjustable gap cell in contact with the electrolyte $\left(0.001 \mathrm{~mol} \cdot \mathrm{dm}^{3} \mathrm{KCl}\right)$. All samples were measured four times at constant $\mathrm{pH}$ value (6.5) with the relative error of $10 \%$. The streaming current method was applied for the determination of the zeta potential. 
For surface elemental analysis there was used X-ray photoelectron spectroscopy. The high vacuum chamber was equipped with source SPECS X-Ray XR50, where Al cathode (1486.6 eV) was used, and with a hemispherical analyzer SPECS PHOIBOS 100 with 5-channels detector.

\section{RESULTS AND DISCUSSION}

SPs were synthesized by redox reaction of $\mathrm{Na}_{2} \mathrm{~S}_{2} \mathrm{O}_{3} \cdot 5 \mathrm{H}_{2} \mathrm{O}$ in oxalic acid and using sodium dodecyl sulphate (SDS) as stabilizer. Sample preparation for DLS analysis was carried out by diluting $100 \mu \mathrm{l}$ of the colloidal solution containing SPs to $1 \mathrm{ml}$ with distilled water. Figure 1A shows size distribution of SPs. SPs are distributed homogeneously within the solution, the mean size of $188.6 \mathrm{~nm}$ with the standard deviation of $26.7 \mathrm{~nm}$. Figure 1B shows the zeta-potential distribution for SPs at their natural $\mathrm{pH}$. The SDS-SPs appear to be negatively charged with the mean value of $(-66.7 \pm 2.1) \mathrm{eV}$, SDS is an anionic surfactant with carbon chain structure [6].
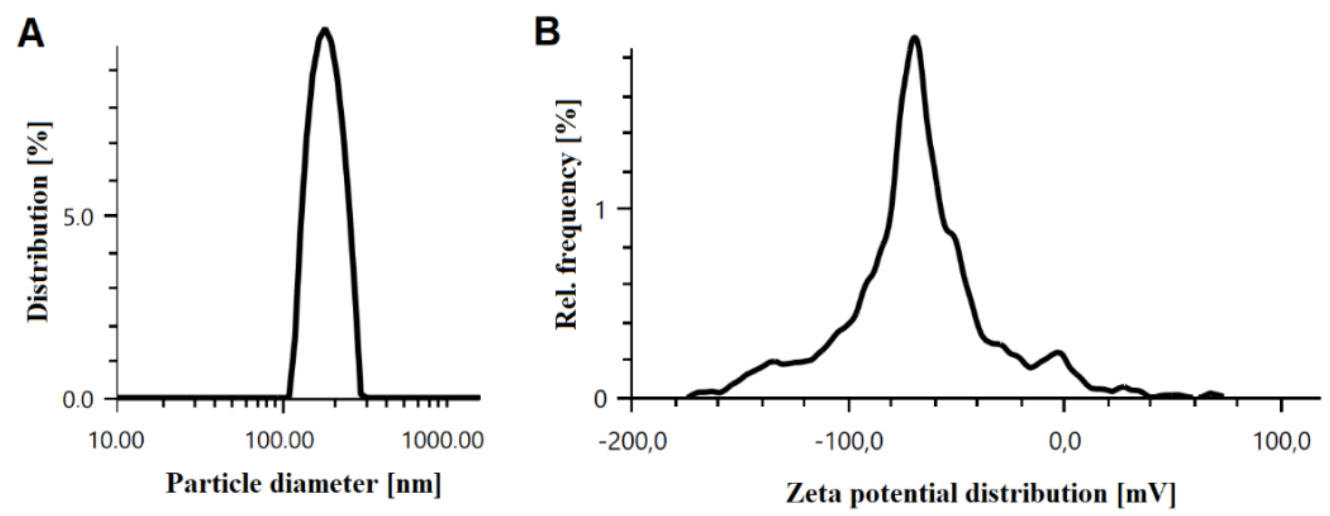

Figure 1 Particle size distribution (A) and zeta potential (B) of freshly prepared SDS-SPs

The tensile test was conducted to evaluate the effect of UV radiation on the mechanical properties of PS foil. The results presented in Figure 2 show the tensile properties of unmodified PS foil are the same in both directions. It is assumed that UV radiation can destroy the polymeric chains [7] and can decrease the tensile strength of polymer foils. The results demonstrated that the tensile strength in transverse direction was not significantly affected by UV radiation. Contrary to longitudinal direction, where the decrease of tensile strength after 30 min of exposure is noticeable.

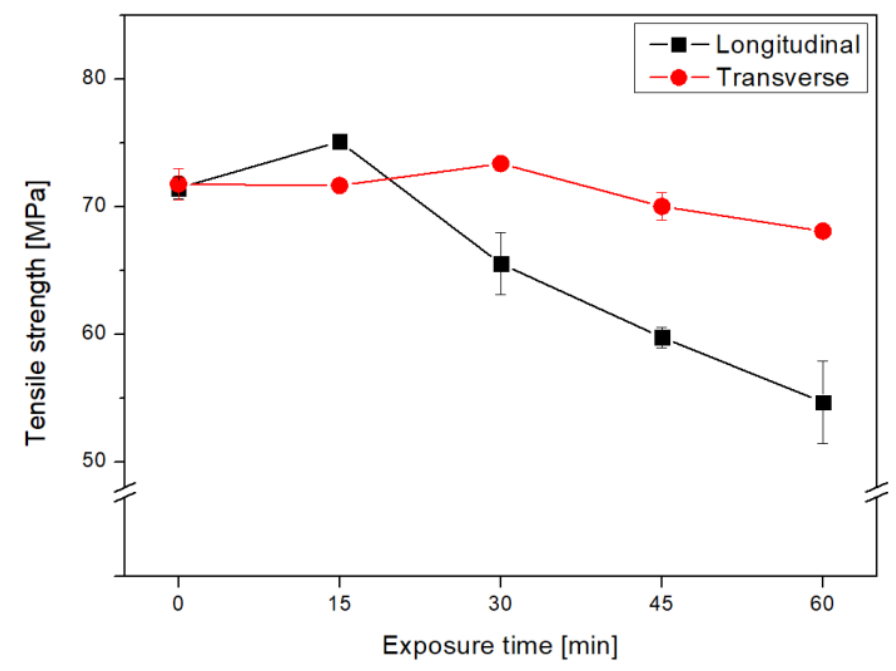

Figure 2 Effect of UV irradiation on tensile strength of PS samples 
The morphology and the size of SDS-SPs after grafting on UV treated PS were characterized using scanning electron microscopy (SEM). The SEM micrographs, presented in Figure 3, show that grafting of SPs on polymer surface results in SPs aggregation with a size of a few micrometers. This effect is probably caused by high surface free energy of PS after UV treatment [8]. However, it is apparent that cysteamine pre-grafting reduces the SDS-SPs aggregation and lead to more homogenous distribution over the polymer surface. It was also found that the amount and change in aggregation of SPs grafted on the polymer surface strongly depends on the UV exposure time. Substrates exposed to UV light by shorter time (15 min) resulted in small number of grafted SPs than substrates modified with times 30-60 min. Otherwise, significant changes in SPs aggregation was observed for substrates modified for a longer time (60 min).
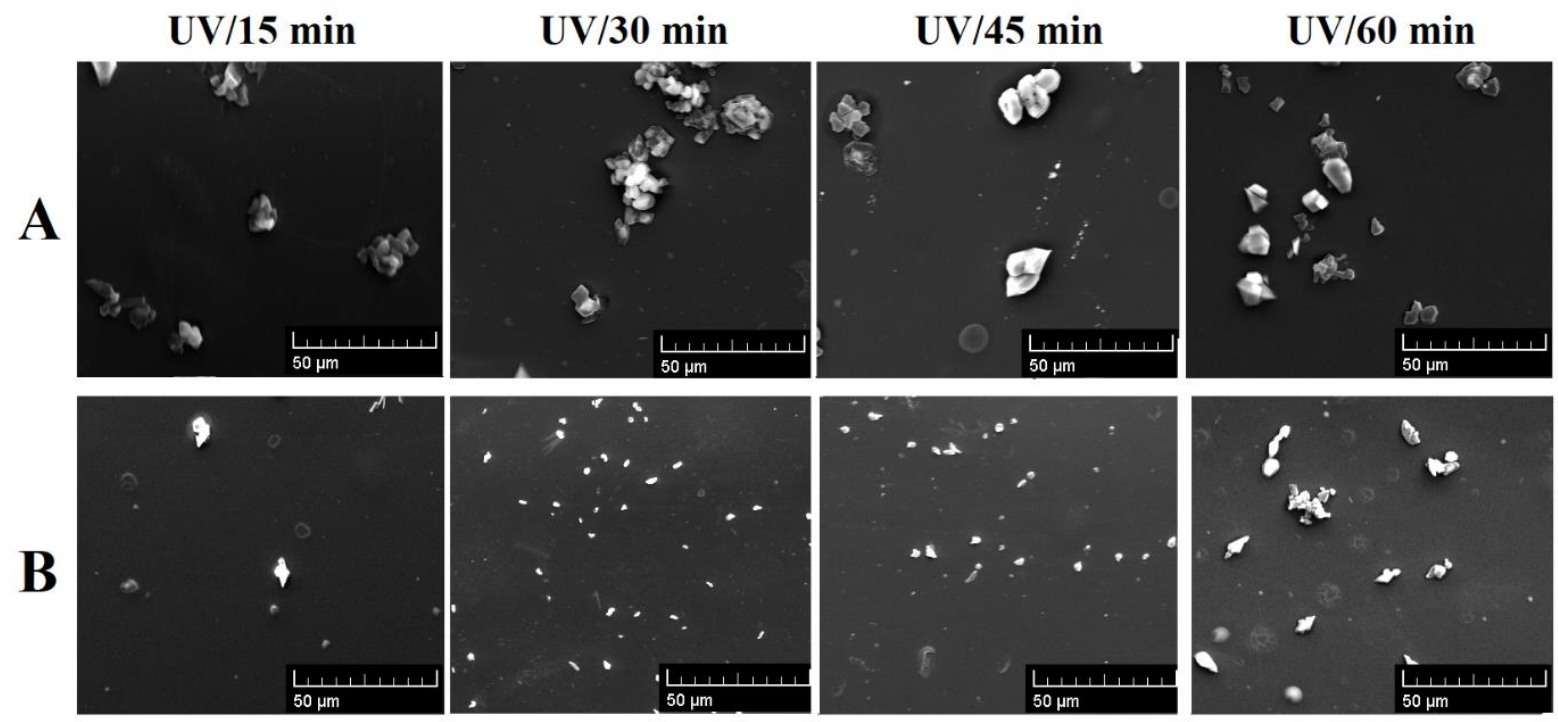

Figure 3 Scanning electron microscopy (SEM) images of SDS-SPs grafted on UV-activated PS surface (A),

UV-activated PS surface pre-grafted with cysteamine (B)

UV treatment is a very clean and effective method for activation of inert polymer surface inducing chemical and structural changes, cleavage of bonds and creation of oxygen-containing and reactive groups [7]. All these processes have a significant effect on contact angle.

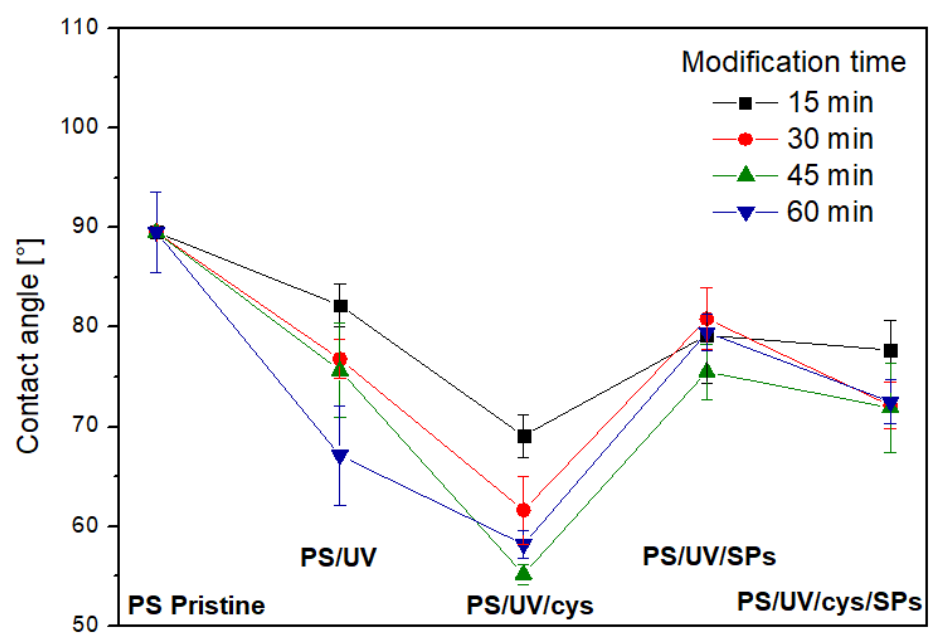

Figure 4 Contact angles of unmodified PS foil (PS Pristine) and after individual steps of modification, after UV exposure (PS/UV) and after subsequent grafting of either cysteamine (PS/UV/cys) or SDS-SPs

(PS/UV/SPs) or both (PS/UV/cys/SPs) 
Figure 4 presents the results of the contact angle measurement of PS foil before and after individual modification steps. All samples after UV exposure exhibit decrease of contact angle caused by creation of oxygen-containing groups on activated surface [9]. The presence of oxygen groups has also been confirmed by XPS determination discussed below. Samples modified by UV for longer time (60 min) had significantly lower values of contact angle $\left(C A=67^{\circ}\right)$ compared with samples exposed for a shorter time $\left(15 \mathrm{~min}, \mathrm{CA}=82^{\circ}\right)$. Contact angle of all samples decreased with increasing UV exposure time. The subsequent cysteamine grafting led to even more hydrophilic surface, on the other hand SDS-SPs grafting caused an increase of contact angle. In comparison with unmodified PS $\left(C A=90^{\circ}\right)$, all modification steps led to more hydrophilic surface.

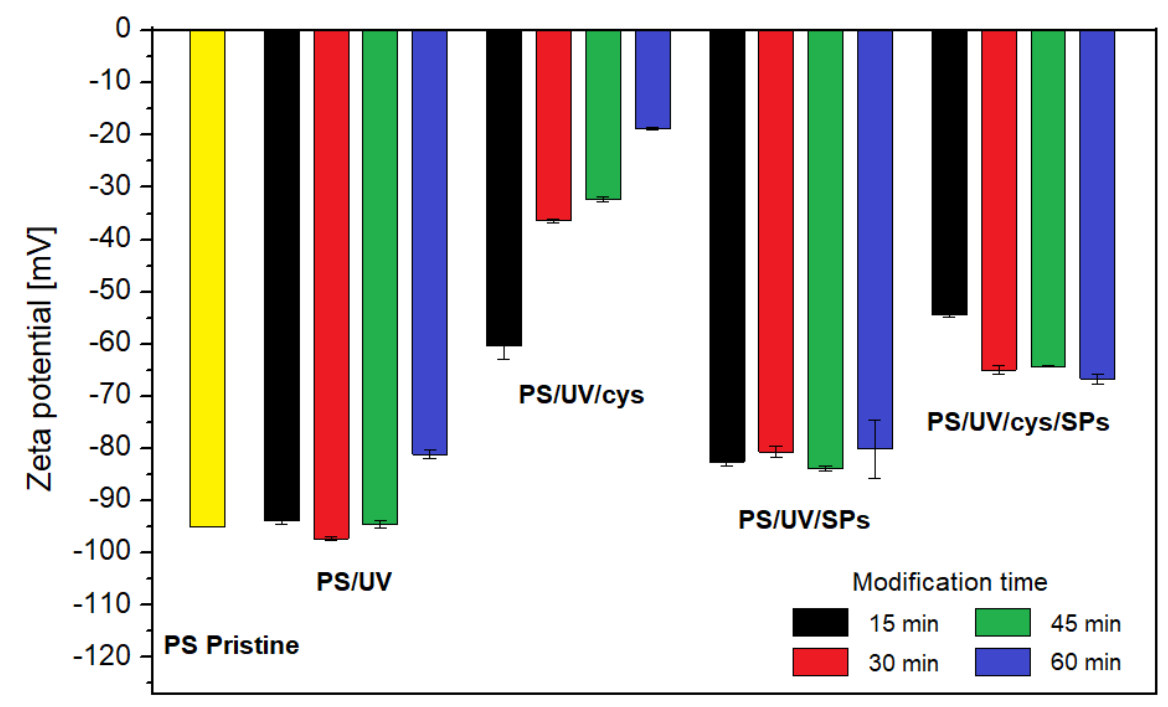

Figure 5 Zeta potential of unmodified PS foil (PS Pristine) and after individual steps of modification, after UV exposure (PS/UV) and after subsequent grafting of either cysteamine (PS/UV/cys) or SDS-SPs (PS/UV/SPs)

or both (PS/UV/cys/SPs)

The zeta potential values before and after individual modification steps are presented in Figure $\mathbf{5}$. As it is evident, the polymer surface properties affect the value of zeta potential and the zeta potential changes after UV treatment and after cysteamine and SDS-SPs grafting. UV radiation causes mild changes of zeta potential due to creation of new polar groups and reactive sites on the surface, most significant change was observed at sample exposed for a longer time $(60 \mathrm{~min})$. The grafting of cysteamine results in a less negative zeta potential, due to the presence of amino- groups, which creates a positive charge on the polymer surface in the presence of electrolyte. Significant differences in values, depending on UV exposure time (increasing zeta potential of PS/UV/cys with increasing time) may be caused by different amount of grafted cysteamine and/or different way of cysteamine bonding to surface (via $-\mathrm{SH}$ or $-\mathrm{NH}_{2}$ group) [10]. All these changes in zeta potential values indicate reliably the successful grafting of cysteamine and SDS-SPs on PS surface.

Table 1 Elemental concentration in at.\% for PS foils as measured by XPS

\begin{tabular}{|c|c|c|c|c|c|}
\hline Sample & C 1s & 0 1s & N 1s & S 2p & Na 1s \\
\hline PS Pristine & 100.0 & - & - & - & - \\
\hline PS/UV (30 min) & 93.1 & 6.9 & - & - & - \\
\hline PS/UV/cys & 89.8 & 7.3 & 1.9 & 1.0 & - \\
\hline PS/UV/SPS & 92.3 & 7.2 & - & 0.4 & 0.1 \\
\hline PS/UV/cys/SPs & 87.6 & 9.0 & 2.0 & 1.3 & 0.1 \\
\hline
\end{tabular}


The elemental composition of the surface of pristine, UV-activated PS for $30 \mathrm{~min}$, and subsequently grafted with cysteamine and/or with SDS-SPs was determined from XPS spectra. The resulting concentrations (in at.\%) are shown in Table 1. UV treatment results in cleavage of bonds in polymer chains and production of free radicals. This causes the oxidation of polymer surface and incorporation of oxygen polar groups, which is detected as an increase of oxygen concentration (from $0 \%$ to $6.9 \%$ ) in XPS spectra. This result was also confirmed by goniometry and electrokinetic analysis. Grafting of cysteamine leads to increase of nitrogen and sulphur on the polymer surface, in the case of SDS-SPs, sulphur and small amount of sodium is observed.

\section{CONCLUSION}

New nanocomposite consisting of polymer (PS) and SDS-capped sulfur particles was prepared. The SPs were synthesized using oxalic acid and sodium thiosulfate in the presence of SDS. A mean particles size was $188.6 \pm 26.7 \mathrm{~nm}$ as determined by DLS. UV treatment, cysteamine and SPs grafting cause changes in surface charge, chemistry and polarity of PS. UV radiation leads to decrease of contact angle and higher hydrophilicity of polymer. The SEM results showed that significant agglomeration of grafting SPs takes place during the process. Cysteamine pre-grafting significantly reduced SPs agglomeration and led to more homogenous distribution over the polystyrene surface. UV exposure time strongly affects the amount and agglomeration of grafted SPs. These findings may be of importance for advance research and development of new composite materials composed of polymers and nanoparticles.

\section{ACKNOWLEDGEMENTS}

This work was supported by GACR under Project 20-01768S, by the Grant Agency of J. E. Purkyně University in Usti nad Labem under project UJEP-SGS-2020-53-007-2, by ERDF/ESF Project „UniQSurf - Centre of biointerfaces and hybrid functional materials"

(No. CZ.02.1.01/0.0/0.0/17_048/0007411) and the Research Infrastructure NanoEnviCz, supported by the Ministry of Education, Youth and Sports of the Czech Republic under Project No. LM2015073.

\section{REFERENCES}

[1] HETEMI, D., PINSON, J. Surface functionalisation of polymers. Chem. Soc. Rev. 2017, vol. 46, p. 5701.

[2] KVITEK, O., REZNICKOVA, A., ZELENAKOVA, A., ZELENAK, V., ORENDAC, M., SVORCIK, V. Immobilization of Fe@Au superparamagnetic nanoparticles on polyethylene. Eur. Polym. J. 2019, vol. 110, p. 56.

[3] GODDARD, J.M., HOTCHKISS, J.H. Polymer surface modification for the attachment of bioactive compounds. Prog. Polym. Sci. 2007, vol. 32, p. 698.

[4] CHAUDHURI, R.G., PARIA, S. Synthesis of sulfur nanoparticles in aqueous surfactant solutions. J. Colloid Interface Sci. 2010, vol. 343, p. 439.

[5] XIE, X., DENG, R., PANG, Y., BAI, Y., ZHENG, W., ZHOU, Y. Adsorption of copper(II) by sulfur microparticles. Chem. Eng. J. 2017, vol. 314, p. 434.

[6] SULEIMAN, M., AL-MASRI, M., AL ALI, A., AREF, D., HUSSEIN, A., SAADEDDIN, I., WARAD, I. Synthesis of nano-sized sulfur nanoparticles and their antibacterial activities. J. Mater. Environ. Sci. 2015, vol. 6, p. 513.

[7] EVE, S., MOHR, J. Study of the surface modification of the PMMA by UV-radiation. Procedia Eng. 2009, vol. 1, p. 237.

[8] LUPÍNKOVÁ, S., KAIMLOVÁ, M., KORMUNDA, M., KOLSKÁ, Z. Chitosan-capped sulfur microparticles grafted on UV-treated PET surface. Surf. Interface Anal. 2021, vol. 53, p. 108.

[9] KASAHARA, T., SHOJI, S., MIZUNO, J., Surface Modification of Polyethylene Terephthalate (PET) by 172-nm Excimer Lamp. J. Japan Inst. Electron. Packag. 2012, vol. 5, pp. 47.

[10] KNAPOVA, T., MATOUSEK, J., KOLAROVA, K., SLEPICKA, P., SICHA, V., TROGL, J., KOLSKÁ, Z. Nanostructured Surface and Antimicrobial Properties of Chemically Modified Polymer Foils. ChemistrySelect. 2019, vol. 4, pp. 4382-4391. 